HER2 in the progression from Barrett's (BE) to OA. This study aimed to clarify expression in this sequence, and to show binding of C6.5, a single chain HER2 targeting human antibody Fv fragment (scFv), to known HER2 expressing OA cell lines in vitro. Efficacy of antibody based therapies can be enhanced by scFv's which penetrate tumours more quickly and demonstrate better tumour: normal tissue specificity.

Methods 33 paraffin embedded oesophageal tissue specimens were selected from patients with squamous $(n=4)$, non-dysplastic $B E$ $(\mathrm{NDBE} ; \mathrm{n}=4)$, low grade dysplasia (LGD; $n=6)$, high grade dysplasia $(\mathrm{HGD} ; \mathrm{n}=8)$ and $\mathrm{OA}(\mathrm{n}=12)$. Sections were immunostained with the automated Oracle Bond system (Leica, UK) for consistency. Staining was then scored by 2 expert pathologists according to the proportion of cells in the tissue staining positively as negative $(<5 \%)$, borderline $(5 \%-10 \%)$ or positive $(>10 \%)$. In phase 2 , binding of C6.5 scFv to the cancer cell lines SKOV-3 (ovarian), OE19, OE-33 (oesophageal) and HT-29 (colon) was identified with flow cytometry using a mouse secondary followed by tertiary anti-mouse FITC. Data were then analysed with FlowJo software.

Results Significant expression of HER2 $(>10 \%$ cells positive according to National Guidelines) was only seen in HGD (25\%) and cancer $(25 \%)$ specimens. Borderline staining $(5 \%-10 \%)$ was seen in LGD (17\%), HGD (13\%) and cancer (8\%). All NDBE and the remaining LGD, HGD and cancer samples were negative. Flow cytometry demonstrated C6.5 binding to SKOV-3, OE19 and OE-33 cells but not HT-29 cells.

Conclusion This study demonstrated that significant HER2 expression is only seen in roughly a quarter of patients with HGD and OA and not in NDBE or LGD. We also found that the HER2 targeting scFv C6.5 binds to breast cancer and OA cell lines but not colon cancer, the negative control. HER2 targeting therapies could therefore be postulated for patients with BE with HGD and OA, and these may be enhanced with scFv's such as C6.5.

Competing interests None declared

\section{PWE-016 EVOLUTION OF ENDOTHERAPY FOR HIGH GRADE DYSPLASIA AND EARLY CANCER IN BARRETT'S OESOPHAGUS: COMPLETING THE AUDIT CYCLE IN A SINGLE CENTRE}

doi:10.1136/gutjnl-2012-302514d.16

M Usman-Saeed,* K Gowland, C Carr, J Buchanan, S Mahmood, H L Smart. Department of Gastroenterology, Royal Liverpool Hospital, Liverpool, UK

Introduction We have previously reported our outcomes for endotherapy in Barrett's oesophagus (BO) patients with high grade dysplasia (HGD) and intra-mucosal adenocarcinoma (IMAC) to this Society. Using Endoscopic Mucosal Resection (EMR) and Argon Plasma Coagulation (APC) in patients unfit for surgery, no procedure related mortality, minimal morbidity but a recurrence rate of $27 \%$ was seen. ${ }^{1}$ We were interested to complete the audit cycle, particularly with the advent of Radiofrequency Ablation (RFA), which has become available since our initial report.

Methods The aim was to re-audit the outcomes of endotherapy for HGD and IMAC in patients with $\mathrm{BO}$ attending our gastroenterology unit and to compare the results to our previous audit in terms of outcome and complications. A retrospective review of patient records using specialised endoscopy and patahology databases was performed. Demographic information, diagnosis, procedural details and subsequent progress was entered into a Microsoft Access database and analysed using Microsoft Excel.

Results A total of 54 patients who underwent endotherapy from January 2005 to December 2011 were identified. The mean age of the group was 71 (range 45-89) years. There were 42 males and 12 females. The underlying diagnosis was HGD in 49 and IMAC in 5.
Patients with focal raised lesions were treated by EMR, while diffuse dysplasia was treated by mucosal ablation (APC or RFA). Eight patients had EMR alone. EMR was followed by APC in six patients and RFA in 20. Five patients had EMR, APC and RFA. Ten patients had RFA and five APC alone. Six patients (11\%) developed an oesophageal stricture requiring dilatation. No bleeding, perforation or procedure related mortality occurred. Over a median follow-up of 18.9 months (range $0-71$ ), two patients (4\%) developed a recurrence of their condition, defined as the reappearance of HGD or IMAC after one or more negative biopsies. Two patients (4\%) had persistent disease. These four patients $(7 \%)$ were referred for surgery and underwent oesophagectomy. Seven patients are still under treatment with RFA, 38 have no dysplasia, four low grade dysplasia with one lost to follow-up. During the follow-up period three patients have died, one in the post-operative period.

Conclusion In our department, endotherapy has evolved with APC being replaced by RFA, often in combination with EMR. This has resulted in a considerable improvement in patient outcomes (7 vs $27 \%$ recurrence/persistent disease), no procedure related mortality and minimal morbidity. Endotherapy is now used as the primary treatment for HGD and IMAC with surgery being reserved as a salvage procedure if no response or disease recurs.

Competing interests None declared.

\section{REFERENCE}

1. Duffy U, et al. Gut 2009;58(Suppl 1):A146.

\section{PWE-017 CAN WE USE BLOOD MARKERS INSTEAD OF ROUTINE CONTRAST SWALLOWS TO PREDICT ANASTOMOTIC DEHISCENCE AFTER OESOPHAGEAL RESECTION?}

doi:10.1136/gutjnl-2012-302514d.17

N Tewari, ${ }^{*}$ J A Catton, N T Welch, S L Parsons. Nottingham University Hospitals NHS Trust, Nottingham, UK

Introduction Resectional surgery for oesophageal cancer is associated with significant risk of anastomotic dehiscence. In the era of enhanced recovery after surgery (ERAS), some anastomotic leaks may not present until after the patient is discharged home. This study aims to identify markers which may predict likelihood of anastomotic dehiscence.

Methods All patients undergoing oesophageal cancer resection from January 2001 to November 2011 were identified from the Upper GI database where data is recorded prospectively. Retrospective review of patient demographics, operation type, radiology, blood results, histology, length of stay and mortality was performed. Blood results of patients with clinical evidence of anastomotic leak and 50 matched controls were collected for the first 10 post-operative days. Results 309 patients (median age 66 years) underwent oesophageal resection during this period. In-hospital mortality occurred in 22 patients (7.1\%). 216 patients $(69.9 \%)$ underwent routine contrast studies and 12 radiological leaks were identified. Two patients with radiological leaks had clinical findings suggestive of anastomotic leak, all were managed conservatively. Anastomotic leakage occurred in a further 20 patients. 13 of these should have undergone contrast swallow at day 7 according to the protocol at the time. Two had contrast swallows at day 7 , which were normal, but subsequently leaked. 16 patients demonstrated clinical deterioration at days 2-7 which prompted either imaging with CT, endoscopy or surgical exploration. Two patients were never fit enough to undergo contrast study. The overall mortality in patients with leak was 5/32 (15.6\%). Results of mean white cell count on days 1 to 9 are given in the Abstract PWE-017 table 1 below. There was a significant difference in white cell count from days 6 to 9 between the control group and leak group. There was a difference in mean CRP in the control group 